\title{
Lumpectomy Versus Mastectomy in Breast Cancer: Comparison of Postoperative Consequences and Treatment Progress
}

\author{
Md. Ashiqur Rahman, " , Farida Arjuman², Shahida Alam³, Mohammad Ibrahim Khalil, \\ Quazi Habibullah ${ }^{5}$, Khandakar A. B. M. Abdullah Al Hasan ${ }^{1}$, Farhana Afroz ${ }^{6}$, \\ Nazneen Naher Aymon ${ }^{7}$ \\ ${ }^{1}$ Department of Surgical Oncology, National Institute of Cancer Research and Hospital, Mohakhali, Dhaka, Bangladesh \\ ${ }^{2}$ Histopathology, National Institute of Cancer Research and Hospital, Mohakhali, Dhaka, Bangladesh \\ ${ }^{3}$ Department of Radiation Oncology, National Institute of Cancer Research and Hospital, Mohakhali, Dhaka, Bangladesh \\ ${ }^{4}$ Department of Surgery, Dhaka Medical College, Dhaka, Bangladesh \\ ${ }^{5}$ Surgery, National Institute of Cancer Research and Hospital, Mohakhali, Dhaka, Bangladesh \\ ${ }^{6}$ Department of Biochemistry, Sheikh Hasina National Institute of Burn and Plastic Surgery, Dhaka, Bangladesh \\ ${ }^{7}$ Pathology, Sir Salimullah Medical College and Mitford Hospital, Dhaka, Bangladesh
}

Email address:

drashique36bd@gmail.com (Md. A. Rahman)

${ }^{*}$ Corresponding author

\section{To cite this article:}

Md. Ashiqur Rahman, Farida Arjuman, Shahida Alam, Mohammad Ibrahim Khalil, Quazi Habibullah, Khandakar A. B. M. Abdullah Al Hasan, Farhana Afroz, Nazneen Naher Aymon. Lumpectomy Versus Mastectomy in Breast Cancer: Comparison of Postoperative Consequences and Treatment Progress. Cancer Research Journal. Vol. 9, No. 1, 2021, pp. 79-84. doi: 10.11648/j.crj.20210901.20

Received: January 26, 2021; Accepted: March 17, 2021; Published: March 30, 2021

\begin{abstract}
Background: Lumpectomy and mastectomy remain the main surgical procedure of breast cancer as a part of treatment as well as management. This study was aimed to compare the early post-operative outcomes of lumpectomy (BCS) versus mastectomy (MRM). Methods: It was an observational study conducted at the Department of Surgical Oncology of National Institute of Cancer Research and Hospital (NICRH), Mohakhali, Dhaka, Bangladesh during the period from October 2016 to January 2019. The study sample consisted of 264 cancer patients who admitted in the hospital. The purposive sampling was done following inclusion criteria. Having collection of data, chi-square test was done to see the association of breast cancer among female patients. Results: Out of 264, 200 (75.75\%) patients underwent mastectomy (MRM) and remaining percentage of patients underwent lumpectomy. The mean age of the patients was 37.69 ( $\mathrm{SD} \pm 10.31$ ) and $44.82(\mathrm{SD} \pm 7.65)$ in lumpectomy and mastectomy group respectively. Wound infection was present among $12.5 \%$ and $24 \%$ lumpectomy and mastectomy patients. Subsequently, seroma was present among $18.75 \%$ and $54 \%$ patients who underwent lumpectomy $(\mathrm{BCS})$ and mastectomy $(\mathrm{MRM})$ respectively which was statistically significant $(\mathrm{P}<0.014)$. Flap necrosis was found only among $16 \%$ mastectomy patients $(\mathrm{P}<0.04)$. There was significant difference in income and education among lumpectomy and mastectomy patients. However, the mean duration of hospital stay was $6.06 \pm 0.85$ days and 17.70 \pm 4.70 days in lumpectomy (BCS) and mastectomy (MRM) group respectively which was statistically significant $(\mathrm{P}<0.05)$. Conclusion: This study showed that lumpectomy $(\mathrm{BCS})$ had early recovery and better post-operative treatment progress than mastectomy (MRM).
\end{abstract}

Keywords: Bangladesh, Flap Necrosis, Lumpectomy, Mastectomy, Seroma 


\section{Introduction}

Breast cancer is the most common cancer in women both in the developed and less developed countries. [17] It is the most frequently diagnosed life-threatening disease faced by women. In less-developed countries, it is considered as the leading cause of death in women. [13] Early diagnosis and effective treatment can reduce the risk of consequences including death. The modern approach to the breast cancer management is multidisciplinary. The cornerstone of breast cancer management is surgical procedure. Of them, the modified radical (Paty) mastectomy (MRM) is the most common surgery for breast cancer management. [17] Alternatively, lumpectomy or Breast conservation surgery (BCS) is also an accepted surgical procedure for early stage of breast cancer which have similar efficacy and effectiveness of treatment over mastectomy. [5, 14, 6] Study also observed that lumpectomy had fewer complications than mastectomy. They observed higher complication rate for mastectomy (4.04\%) than lumpectomy (1.74\%) during 30 days' postoperative period. [3] Hence, lumpectomy is mostly recommended surgery for breast cancer over mastectomy. In Vienna consensus 2015 also mentioned that breastconservation surgery (BCS) or lumpectomy is the standard care even in cases of multifocal or multicentric disease. [7]

However, the common complications includes wound infection, seroma, hematoma and skin flap necrosis within 30 postoperative days of breast surgery. [11] Of them, the most frequent-complication is wound infection. A study compared the frequency of infection and found higher infection among mastectomy (4.34\%) than lumpectomy patients $(1.97 \%)$ [4]Another study also showed similar results. They found wound infection rates in mastectomy versus lumpectomy were $0.81 \%$ and $0.28 \%$ respectively. [3]

Along with wound infection, seroma formation is also a significant complication followed by breast surgery. Iram Bokhary et al. validated by their study that seroma was the most common complication of breast surgery. [2] A study found around $3 \%$ of seroma after mastectomy. [18] Another study also compared the prevalence of seroma between mastectomy and lumpectomy. They observed about 29\% in mastectomy (MRM) and 18\% in lumpectomy (BCS) [15]

Epidermolysis or flap necrosis is described as having some degree of skin necrosis. It is another troublesome complication following breast surgery which is mostly observed after mastectomy. A study found $6 \%$ to $18 \%$ flap necrosis in mastectomy (MRM) where as $0 \%$ in lumpectomy. [2] In addition, hematoma is another common complication of mastectomy. Though widespread use of electrocautery significantly reduces the incidence of hematoma formation this complication continues to occur in $2 \%$ to $10 \%$ of mastectomy cases. [1] Another study showed that hematoma occurs $13 \%$ in lumpectomy (BCS) and $29 \%$ in mastectomy (MRM) group. [16] Subsequently, early arm oedema is said to occur about half of the patients after axillary dissection. The majority of patients undergo some degree of oedema particularly due to lack of awareness Study showed a certain percentage of lymphedema (28\% and $27.8 \%$ ) following breast surgery. [19] In Bangladesh, breast cancer is the most common cancer among women. Nearly 15,000 cases of breast cancer being are detected in each year, of which 16.9 percent die in this country. [16] Like other countries, breast cancer patients are being managed mainly by mastectomy in Bangladesh. However, recently lumpectomy (BCS) is being popular as effective surgical procedure for management of breast cancer particularly due to lower risk of complication and early recovery. Unfortunately, there is no specific comparative research conducted in Bangladesh. So, authors felt the necessity to conduct such study to interpret potential benefits of lumpectomy over mastectomy with evidence.

\section{Materials and Methods}

The study related socio-demographic and socio-economic data were collected from patients with confirm breast cancer who admitted at Department of Surgical oncology of National Institute of cancer Research and Hospital (NICRH), Mohakhali, Dhaka, Bangladesh, from October 2016 to Jan 2019. A total of 264 female patients were purposively selected during the study period. As NICRH is the national level specialized hospital, patients were referred from different geographic locations covering all divisions of Bangladesh. All selected patients were registered at the institute before start of treatment. Having admitted, the study was conducted with the permission of concerned authority of the institute. A semi-structured questionnaire was developed for data collection. The study purpose was properly shared with respondent and a concrete written consent of each respondent was taken prior to initiation of face to face interview. Respondents had the opportunity to accept or reject the study purpose. However, the socio-demographic and socio-economic data includes age, income, education, marital status, family history of breast cancer, history of oral contraceptive pill. Each patient was handled with proper line of treatment such as history, examination, investigation, treatment with follow up evaluation. However, for surgical procedure, patients were grouped into two -1 . Mastectomy, a broad group who fulfilled standard criteria for mastectomy. 2. Lumpectomy, a small group following inclusion criteria such as age below 50 years, small size $(<4 \mathrm{~cm})$, unicentric and peripherally located tumour. The remaining patients were excluded from the study who admitted with stage IV disease, recurrent and ulcerated/fungating mass. After successful operation, each patient received a broad spectrum antibiotic, analgesic and electrolytes as per need and each patient was carefully and daily followed up at regular basis for first seven days and then periodic follow-up up to 30 days and recorded the treatment progress and complications between two groups. All relevant data were recorded using excel sheet and finally transferred to SPSS IBM version 2016 for analysis. Frequency distribution and Chi-square test was done to interpret the findings with level of significance. 


\section{Results}

A total of 264 patients were enrolled for the study. Of them, 64 (24.24\%) underwent lumpectomy as they had small size, unicentric peripheral tumour. The remaining respondents $200(75.75 \%)$ underwent mastectomy as they did not meet the lumpectomy criteria (Figure 1).

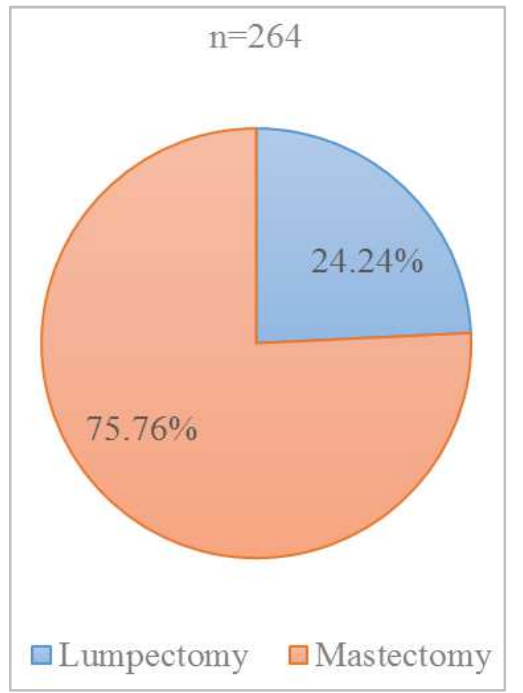

Figure 1. Distribution of patients according to type of surgery.

The mean age for lumpectomy and mastectomy was 37.69 and 44.82 years respectively. All lumpectomy patients $(100 \%)$ were married where as a portion of mastectomy patients were divorced (4.0\%) and widowed (6.0\%) respectively. The remaining $(90.0 \%)$ mastectomy patients were married. There was no unmarried breast cancer patients. However, the mean body mass index (BMI) was within the normal range for both groups which as 23.65 and 24.13 respectively. Most of the mastectomy patients were belongs to poor and middle income group $(28.0 \%$ and $60.0 \%)$ whose monthly family income range was below 10000 BDT. to 25000 BDT. Surprisingly, most lumpectomy patients (75.0\%) were belongs to rich income group whose monthly family income was more than 25000 BDT. Subsequently, most of the mastectomy patients had primary (34\%) and secondary $(42 \%)$ level education whereas majority of lumpectomy patients were higher secondary $(>56 \%)$ and graduate and above $(>18 \%)$ level educated. Both lactating and nonlactating mother faces breast cancer. Of them, mastectomy (92.0\%) was higher than lumpectomy (68.8\%) among lactating mothers. Opposite findings were observed among non-lactating mothers. Along with socio-economic, sociodemographic factors, certain behavioral factors and physiological conditions were also evaluated. To know the effect of hormone on developing breast cancer as behavioral factor, history of taking oral contraceptive pills (OCP) were analyzed. Most of the lumpectomy patients received OCP $(>87 \%)$. Subsequently around half of the mastectomy patients had OCP history $(42 \%)$. The difference was statistically significant $(\mathrm{P}<0.001)$. Surprisingly, more than half of the mastectomy patients $(58 \%)$ did not take OCP. (Table 1).

Table 1. Distribution of socio-demographic and behavioral factors according to types of surgery.

\begin{tabular}{|c|c|c|c|}
\hline Variables & Lumpectomy & Mastectomy & P value \\
\hline Mean age in years with SD & $37.69 \pm 10.31$ & $44.82 \pm 7.65$ & 0.001 \\
\hline Mean BMI with SD & $23.65 \pm 1.29$ & $24.13 \pm 1.67$ & \\
\hline \multicolumn{4}{|l|}{ Monthly family income in BDT. } \\
\hline Middle income (10000-25000) & $16(25.0 \%)$ & $120(60.0 \%)$ & \\
\hline $\operatorname{Rich}(\geq 25000)$ & $48(75.0 \%)$ & $24(12.0 \%)$ & \\
\hline \multicolumn{4}{|l|}{ Family history of breast cancer } \\
\hline No & $48(75.0 \%)$ & $160(80.0 \%)$ & \\
\hline \multicolumn{4}{|l|}{ Education } \\
\hline Primary (0-5 years of schooling) & $4(6.2 \%)$ & $68(34.0 \%)$ & 0.004 \\
\hline Secondary (6-10 years of schooling) & $12(18.8 \%)$ & $84(42.0 \%)$ & \\
\hline Higher secondary (11-12 years of schooling) & $36(56.3 \%)$ & $32(16.0 \%)$ & \\
\hline Graduate and above ( $>12$ years of schooling) & $12(18.7 \%)$ & $16(8.0 \%)$ & \\
\hline \multicolumn{4}{|l|}{ Marital status } \\
\hline Married & $64(100 \%)$ & $180(90.0 \%)$ & 0.563 \\
\hline Widowed & $0(0 \%)$ & $12(6.0 \%)$ & \\
\hline \multicolumn{4}{|l|}{ Lactation status } \\
\hline Currently lactating & $44(68.7 \%)$ & $184(92.0 \%)$ & 0.004 \\
\hline Currently non-lactating & $20(31.3 \%)$ & $16(8.0 \%)$ & \\
\hline \multicolumn{4}{|l|}{ History of oral contraceptive pills (OCP) } \\
\hline Yes & $56(87.5 \%)$ & $84(42.0 \%)$ & 0.001 \\
\hline No & $8(12.5)$ & $116(58.0 \%)$ & \\
\hline
\end{tabular}

Chi-square test also shows more post-operative complication for mastectomy than lumpectomy. The mastectomy patients experienced with would infection $(24 \%)$, seroma $(54 \%)$, flap necrosis $(16 \%)$, early arm oedema
(40\%) and hematoma (14\%). On the other hand, lumpectomy patients did not face flap necrosis $(\mathrm{P}<0.05)$ and the other mentioned complication was lower than mastectomy. The duration of hospital stay for lumpectomy was less than 7 days 
whereas for mastectomy, it was more than 17 days. (Table 2).

Table 2. Distribution of patients according to the early postoperative complications and mean duration of hospital stay ( $n=264)$.

\begin{tabular}{|c|c|c|c|}
\hline Variables & Lumpectomy & Mastectomy & P value \\
\hline \multicolumn{4}{|l|}{ Wound infection } \\
\hline Yes & $8(12.5 \%)$ & $48(24.0 \%)$ & 0.327 \\
\hline No & $56(87.5 \%)$ & $152(76.0 \%)$ & \\
\hline \multicolumn{4}{|l|}{ Seroma formation } \\
\hline Yes & $12(18.7 \%)$ & $108(54.0 \%)$ & 0.014 \\
\hline No & $52(81.3 \%)$ & $92(46.0 \%)$ & \\
\hline \multicolumn{4}{|l|}{ Flap necrosis } \\
\hline Yes & $0(0.0 \%)$ & $32(16.0 \%)$ & 0.040 \\
\hline No & $64(100 \%)$ & $168(84.0 \%)$ & \\
\hline \multicolumn{4}{|l|}{ Arm oedema } \\
\hline Yes & $12(18.7 \%)$ & $80(40.0 \%)$ & 0.159 \\
\hline No & $52(81.3 \%)$ & $120(60 \%)$ & \\
\hline \multicolumn{4}{|l|}{ Hematoma } \\
\hline Yes & $4(6.2 \%)$ & $28(14.0 \%)$ & 0.523 \\
\hline No & $60(93.7 \%)$ & $172(86.0 \%)$ & \\
\hline Mean days of hospital stay with SD & $6.06 \pm 0.85$ & $17.70 \pm 4.70$ & 0.001 \\
\hline
\end{tabular}

\section{Discussion}

Out of 264 patients with breast cancer, $24.3 \%$ underwent lumpectomy and the remaining portion of patients underwent modified radical mastectomy (MRM). The criteria for lumpectomy is generally the age below 50 years with small size unicentric tumour which is providing clues to get early screening and diagnosis. In our study, this percentage indicates the level of awareness on breast cancer screening in early stage. The benefits of early detection and treatment is huge such as less sufferings, less cost, less chance of spread and less chance of complication following surgical treatment.

We observed the average age for lumpectomy and mastectomy was 37.69 and 44.82 years respectively. Different studies also shows nearly similar results on age distribution $[8,12]$ Male breast cancer is very rare and we did not find any male patients during our study period. In case of income group, most lumpectomy patients were solvent (75\%) than mastectomy $(\mathrm{P}<0.001)$. Alternatively, most of the mastectomy patients were poor and middle income group whose monthly family income were less than BDT. 25000 $(88 \%)$. So, poor income group might be less aware than rich income group or poor income group patients seek care in late stage. Subsequently, most lumpectomy patients $(75 \%)$ were higher secondary to above level educated. The opposite findings were observed among mastectomy patients who were mostly secondary to below level (76\%). In Bangladesh, higher educated person have higher income opportunity and they are more aware than less educated person. In our study, we observed that those who were less educated and poor to middle income group, they usually choose mastectomy to lower the surgery cost. Unfortunately, they suffered more and spent more costs due to post-operative complications. However, in our study, there was no unmarried breast cancer patient. But we found divorced (4\%) and widowed (6\%) patients who underwent mastectomy. However, the rest of the patients were married irrespective of surgical intervention. We also analyzed data on use of oral contraceptive pill (OCP) to see the hormonal influence on breast cancer and we found that more than $87 \%$ lumpectomy patients took OCP whereas it was less than half (42\%) for mastectomy patients $(\mathrm{P}<0.001)$. As most mastectomy patients used other methods of family planning, $58 \%$ did not take OCP. This findings may indicate the hormonal influence on unicentric peripheral breast cancer. This findings can be the potential clues to researcher for further study. All respondents' underweight surgery and carefully monitored in post-operative words at regular interval. In order to keep records on consequences and complication following surgery with antibiotics and analgesics, each patient was carefully asked, observed and examined for wound healing or complication. However, in our study, we observed $12.5 \%$ and $24 \%$ wound infection among lumpectomy (BCS) and mastectomy (MRM) respectively. A study carried out by Rostein at al. and Somers et al. showed $2 \%-14 \%$ and $3 \%-19 \%$ wound infection among lumpectomy and mastectomy respectively which are nearly similar to our study. $[9,10]$ Our study demonstrated that more than half of the patients (54\%) developed seroma who underwent mastectomy. On the other hand, less than one fifth $(<19 \%)$ of the lumpectomy patients faced seroma indicates higher chance of post-operative complication in modified radical mastectomy (MRM) than lumpectomy (BCS).

Kumar et al. clearly declared the incidence of seroma can occurs after mastectomy (MRM) at a range of $3 \%$ to $85 \%$ which has validated our findings. Though another study observed $18 \%$ and $29 \%$ seroma among lumpectomy and mastectomy patients respectively which was lower than our study, similar results were on surgical procedure that means they also found more seroma among mastectomy patients than lumpectomy. [15] In this study, flap necrosis was observed only among mastectomy (MRM) patients (16.0\%). Another study also showed similar results which was $6 \%$ $18 \%$ flap necrosis followed by mastectomy and zero necrosis followed by lumpectomy. [2] Subsequently, we observed another complication named lymphedema which was $18.7 \%$ and $40.0 \%$ among lumpectomy and mastectomy respectively. A study also showed almost similar results which was $27.8 \%$ 
and $28.0 \%$ respectively. Similarly, we found a portion of post-operative patients faced hematoma. It was also higher among mastectomy (14.0\%) than lumpectomy (6.2\%). Another study generally depicted that hematoma can occur among $2 \%$ to $10 \%$ of breast surgery patients. Though our study exceed the range for mastectomy, we argue for this rate due to patient's physical condition or immunity, surgical procedure or cross contamination inside or outside of Operation Theater.

Each patient was discharged from the hospital following proper evaluation. In our study, patients were physically fit for discharge in a wide variety of duration of hospital stay. However, the mean duration of hospital stay was 6.1 days for lumpectomy and 17.7 days for mastectomy.

\section{Limitation of the Study}

We conducted this study with several limitations. We did not take detailed personal history such as smoking behavior, duration and exposure to radiation and sunlight, diabetes, hypertension and other co-morbidities. We did not take occupational history. Though we took BMI and family history of breast cancer, we did not take number of affected family members and size of family. We excluded complicated cases to simplify the study. We did not mention geographic distribution of breast cancer. The above mentioned limitations would potentially help researchers for further study.

\section{Conclusion}

Surgery is the cornerstone of the multidisciplinary management of breast cancer. Recently, the surgical treatment of breast cancer has undergone a paradigm shift from radical mastectomy (MRM) to lumpectomy or breast conservation surgery (BCS). Along with community awareness on early screening and choice of surgery, BCS would effectively reduce post-operative surgical complication, could help rapid cure. It would also reduce costs and consequences; and improve patient's satisfaction.

\section{Ethical Approval}

The ethical approval for this study was taken from National Institute of Cancer Research and hospital (NICRH), Mohakhali, Dhaka, Bangladesh. After submission of protocol with objectives, approval was taken with proper instruction.

\section{Acknowledgements}

The authors are grateful to the authority of NICRH to provide approval and data collection opportunity. All authors are gratefully acknowledged the best cooperation of patients in spite of their morbid condition.

\section{Conflict of Interest}

All authors declare that they have no conflict of interest.

\section{Funding}

There were no funding sources for this study.

\section{Author's Contribution}

MAR and MAS designed this study. MAR collected data from patients before and after surgery. MAR and MAS analyzed the data and prepared this manuscript. FA, MIK, $\mathrm{QH}, \mathrm{KAH}, \mathrm{FA}, \mathrm{NNA}$ revised the manuscript and provided technical inputs. MAR and MAS re-reviewed and finalized the manuscript. All authors approved the final version.

\section{References}

[1] Angelique, FV \& Lisa, AN., 2007, 'Complications in breast surgery’, SurgClin N Am, vol. 87, pp. 431-451.

[2] Bokhari I., Mehmood, Z., Nazeer, M., \& Khan, A. 2010,'Early complications of mastectomy with axillary clearance in patients with stage II and III carcinoma breast' Journal of Surgery Pakistan (International, vol. 15 (4), pp. 182-185.

[3] Chatterjee, A., Pyfer, B., Fisher, C., Czerniecki, B., Tchou, J., \& Rosenkranz, K. 2015, 'Early postoperative outcomes in lumpectomy versus simple mastectomy', Journal of Surgical Research, vol. 198, pp. 143-148.

[4] El-Tamer, MB., Ward, BM., Schifftner, T., Neumayer, L., Khuri, S., \& Henderson, W. 2007,'Morbidity and mortality following breast cancer surgery in women: National benchmarks for standards of care', Ann Surg, vol. 245 (5), pp. 665-671.

[5] Fisher, B., Anderson, S., Bryant, J., 2002,'Twenty year follow-up of a randomized trial comparing total mastectomy, lumpectomy, and lumpectomy plus irradiation for the treatment of invasive breast cancer', N Engl J Med, vol. 347, pp. $1233-1241$.

[6] Clarke, M., Collins, R., Darby, S., Devies, C., Godwin, J., Hicks, C., et al 2005 'Effects of radiotherapy and of differences in the extent of surgery for early breast cancer on local recurrence and 15-years survival: an overview of the randomised trials' Lancet, vol. 366 (9503), pp. 20872106.

[7] Gnant, M., Thomssen, C., \& Harbeck. St. Gallen/Vienna 2015, 'A brief summary of the consensus discussion', BreastCare, vol. 10, pp. 124-130.

[8] Rahman, MM., Ahsan, MA., Monalissa, NN., Rahman, K. 2014, 'Influence of Socioeconomic Status and BMI on the Quality of Life after Mastectomy in Bangladeshi Breast Cancer Patients in a public Hospital', Japanese Journal of Clinical Oncology, vol. 44 (12), pp. 1150-1157.

[9] Roststein, C., Farguson, R., Cumming, K. M., Piedmonte, M. R., Lucey, J., \& Banish, A. 1992,'Determinants of clean surgical wound infections for breast procedures at an oncology center', Infect Control Hosp Epidemiol, vol. 161 (4), pp. 207-214.

[10] Somers, RG., Jablon, LK., Sandler, GI., Rosenblatt, NK 1992,'The use of closed suction drainage after lumpectomy and axillary node dissection for breast cancer: A prospective randomized trial', Ann Surg, vol. 215, pp. 146-149. 
[11] Sorensen, L. T., Horby, J., Frilis, E., Pilsgaard, B., \& Jorgensen, T. 2002,'Smoking as a risk factor for wound healing and infection in breast cancer surgery', Eur J Surg Oncol, vol. 28 (8), pp. 815-820.

[12] Tiwari, S., Reeni, M., Trichal, VK., Nigam, RK., Rai, A., Balani, S. 2015, 'Breast Cancer: Correlation of Molecular Classification with Clinicohistopathology,'Scholars Journal of Applied Medical sciences', vol. 3 (2G), pp. 1018-1026.

[13] Torre, LA., Bray, F., Siegel, L., Ferlay, J., Loret-Tieutel, J., Jemal, A. 2015, 'Global cancer statistics, 2012, CA: A Cancer J Clin, vol. 65 (2), pp. 87-108.

[14] Veronesi, U., Cascinelli, N., Mariani, L., et al. 2002,'Twenty year follow-up of a randomized study comparing breast conserving surgery with radical mastectomy for early breast cancer', N Engl J Med, vol. 347 (16), pp. 1227-1232.
[15] Vinton, AL., Traverso, LW., \& Jolly, P. C. 1991,'Wound complication after modified radical mastectomy compared with tylectomy with axillary lymph node dissection', Am J Surg, vol. 161 (5), pp. 584-587.

[16] WHO 2016, Retrieved August 20, 2016, from WHO Website: http://www.who.int/cancer/detection/breastcancer/en/index1.ht $\mathrm{ml}$

[17] Thompsom, AM. 1999, 'How I do it. Axillary node clearance for breast cancer' J R Coll Surg Edinberg, vol. 44 (2), pp. 111116.

[18] Kumar, S., Lal, B and Misra, MC, 1995, 'Post mastectomy seroma; a new look into the aetiology of an old problem', world J R coll Surg Edin, vol, 40, pp. 492-496.

[19] Ozaslan, C., \& Kuru, B. 2004, 'Lymphedema after treatment of breast cancer', am J Surg, vol. 187, pp. 69-72. 